\title{
Radiation characteristics of a coaxial waveguide with eccentric inner conductor for application in hyperthermia and microwave reflex therapy
}

\author{
R. Herschmann ${ }^{1,2}$ and O. Büchel ${ }^{3}$ \\ ${ }^{1}$ Leibniz Universität Hannover, Institute of Radiofrequency and Microwave Engineering, Appelstraße 9a, 30167 Hannover, \\ Germany \\ ${ }^{2}$ Smart Devices GmbH \& Co. KG, Schönebecker Allee 2, 30823 Garbsen, Germany \\ ${ }^{3}$ Fuba Automotive GmbH \& Co. KG, Tec Center, 31162 Bad Salzdetfurth, Germany
}

\begin{abstract}
This paper examines the radiation characteristics of a contact emitter conceived for application in hyperthermia and microwave reflex therapy. It is important to analyse the distribution of power density in the near field area, as the radiator's therapeutic sphere of activity is localized here. The contact emitter is a coaxial radiator with an eccentric course of the inner conductor. According to Huygens principle, a theoretical view of the near field radiation characteristics is made by determining the equivalent current densities in the emitter aperture. It is shown that by an eccentric shift of the inner conductor, an almost isotropic near field radiation pattern and power density can be achieved. For this, the electromagnetic field in the emitter aperture is determined by using a Bipolar coordinate system. This calculation considers only the fundamental TEM mode of the contact emitter. Besides the theoretical results near and far fields are simulated using the programme system Ansoft HFSS.
\end{abstract}

\section{Introduction}

Hyperthermia is a technique used in the medical treatments of cancer and other medical therapy. Tumors are heated to therapeutic temperatures (invasive $T>42.5^{\circ} \mathrm{C}$, non invasive $T=40^{\circ} \mathrm{C}-42^{\circ} \mathrm{C}$ without overheating the surrounding normal tissue. Non invasive hyperthermia is used often in combination with chemotherapy and radiation therapy for higher complete response compared to conventional therapies alone. Microwave reflex therapy is a relatively new working method in medicine. High frequency, electromagnetic energy is concentrated on a closely restricted, biological active point by use of a contact emitter. The emitter is put directly on the skin. It provides a type of non invasive acupuncture. The

Correspondence to: R. Herschmann

(herschmann@hft.uni-hannover.de) contact emitter introduced in this paper was developed for use at the skin surface area.

For application within the medical area the emitter aperture must be as small as possible in order to ensure a defined irradiation of the cancerous tissue or biological active point. Furthermore, the electromagnetic field must be isotropic in direct proximity to the effect. A broad emitter bandwidth is necessary in order to adapt the frequency of radiotherapy individually in the examined range from $f=(8-20) \mathrm{GHz}$. The emitter must rest upon the skin well and the impedance of the contact emitter has to be adapted to the human tissue over a broad frequency range. The described requirement profile is fulfilled, to a large extent, by a coaxial radiator. These radiators have long found various applications within the area of non destructive material testing (see Mosig et al., 1981; Bakhtiari et al., 1994), as well as the determination of the dielectric properties of biological substances (see Stuchly and Stuchly, 1980; Stuchly et al., 1982). However, the directional characteristic of a coaxial emitter has a dip in axial direction. Therefore, the desired concentration of radiation intensity cannot be achieved in the near field. A simple possibility for a remedy offers an eccentric shift of the inner conductor in the emitter aperture. The influence of the inner conductor's eccentricity on the radiation pattern can be illustrated with the following thought experiment. One can understand the inner conductor as a dipole from which a radiation dip in axial direction follows. The consequence of an eccentric shift of the inner conductor is a misalignment of the radiation minimum from the symmetry axis, whereby the radiation pattern of the complete arrangement is influenced.

In this publication, the theory for analysing the near field of the radiator described above is based upon the determination of equivalent current densities in the emitter aperture, according to Huygen's principle. The theoretical investigations serve two purposes: to acknowledge the thought experiment; and to support the later presented results simulated by the programme system Ansoft HFSS within the near field

Published by Copernicus Publications on behalf of the URSI Landesausschuss in der Bundesrepublik Deutschland e.V. 
(a)

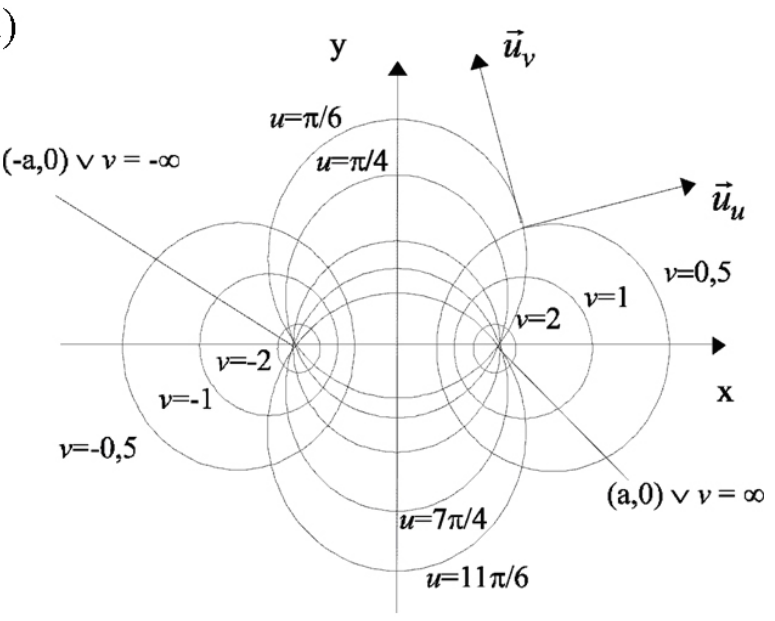

(b)

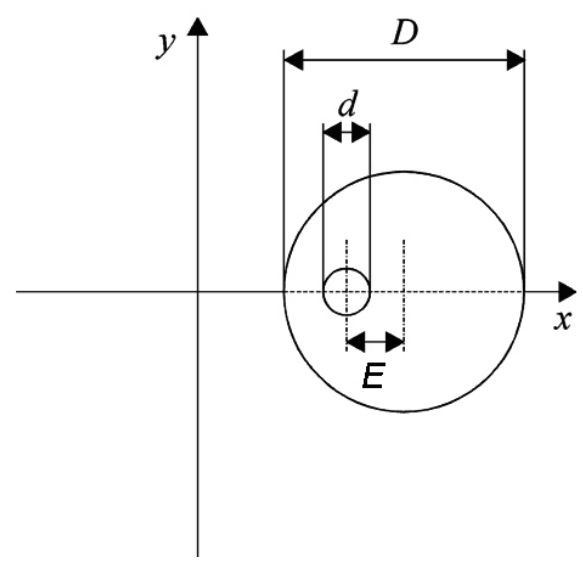

Fig. 1. Interrelation between the geometry of the Bipolar coordinate system (a) and the geometry of the contact emitter (b).

area. To determinate the equivalent sources only the fundamental TEM mode is considered for propagation inside the line. In the mathematical analysis, higher order modes are not considered. Besides the radiator (i.e. geometrical dimensions, dielectric characteristics), the mathematical model describing the radiation behaviour contains the infinitely expanded, semi-infinite space in front of the aperture filled with an isotropic homogenous material. Additionally, the far field radiation characteristics are represented as a result of the analytical approach and simulation.

\section{Theory}

With the analysis of conventional antennas, one is usually interested in the far field characteristics. However, the therapeutic sphere of activity of the contact emitter for medical applications is situated in the near field area. The therapeutic area for local hyperthermia and the biological active points stimulated by microwave reflex therapy are located at the skin surface, which can be $2-12 \mathrm{~mm}$ thick (see Kalantaewskaja, 1972). Therefore, an analysis of near field characteristics is necessary.

The human body consists of a layered tissue with different electrical characteristics. During the irradiation of electromagnetic energy, reflections occur at the transitions between the individual layers. In the following, the layering of tissue is neglected and a homogenous skin tissue with an infinite expansion in the illuminated semi-infinite space is assumed.

In order to predict the radiation behaviour of the radiator exactly, the field equations of the sub ranges feeder, transition zone and infinite space have to be solved by using boundary conditions. In some publications, approaches and methods for the solution of similar problems were already represented. Sumbar et al. presented solutions for field equations relying on the finite element method (see Sumbar, 1991). However, due to the approximated boundary conditions at the sub zone transitions, the solution can only be approximated. In Mosig et al. (1981) and Nevels and Wheeler (1989), the field equations for special coaxial radiators were solved by applying the method of moments. In Maloney et al. (1990), the FD-TD method (finite-difference time-domain method) was used as a numerical solution for radiation problems in the time domain. The procedures mentioned were used exclusively for far field calculation and contain a multitude of neglects. In the theoretical part of this work, the radiation behaviour is determined with the help of the equivalent current densities in the emitter aperture, according to Huygen's principle. The calculation is executed under the following prerequisites:

1. Inside the line, the TEM fundamental mode propagates.

2. The analysis does not consider higher order modes stimulated in the emitter aperture.

3. Energy is radiated only into the semi-infinite space in front of the aperture.

4. The radiator is lossless.

A Bipolar coordinate system is used in order to determine the field distribution in the aperture of the radiator. In this coordinate system, the conductor surface coincides with a coordinate surface in each case.

In Fig. 1, the Bipolar coordinate system $(u, v, z)$ is compared to the geometry of the emitter aperture to explain the interrelations. For a better orientation, the Cartesian coordinate system is projected onto the Bipolar coordinate system in Fig. 1. The geometry of the emitter aperture is characterised by the inside diameter $D$ of the outer conductor, the outside diameter $d$ of the inner conductor and by the eccentricity $E=e \cdot D / 2$ of the inner conductor in the emitter aperture. For instance, if the eccentricity factor $e=0.5$ the centre of the inner conductor is positioned between the centre and the outside margin of the outer conductor. 
2.1 Calculation of the field distribution in the emitter aperture

Because of the assumptions given above, only transversal field components exist in the emitter aperture. These components can be written in Bipolar coordinates as follows:

$$
\begin{aligned}
& \underline{\boldsymbol{E}}=\underline{\boldsymbol{E}}_{v}=\underline{\boldsymbol{e}}_{v}(u, v) \cdot e^{-\underline{\gamma}_{z} \cdot z} \\
& \underline{\boldsymbol{H}}=\underline{\boldsymbol{H}}_{u}=\underline{\boldsymbol{h}}_{u}(u, v) \cdot \mathrm{e}^{-\underline{\gamma}_{z} \cdot z}
\end{aligned}
$$

If this approach is inserted in Maxwell's second equation, $\operatorname{rot} \underline{\boldsymbol{E}}=-\mu \partial \underline{\boldsymbol{H}} / \partial t$, then the following conditions result on the assumption of a time harmonic dependence:

$\operatorname{rot} \underline{\boldsymbol{e}_{v}}(u, v)=0$ and $\underline{\gamma}_{z} \cdot \boldsymbol{u}_{z} \times \underline{\boldsymbol{e}}_{v}(u, v)=j \omega \mu \cdot \underline{\boldsymbol{h}}_{u}(u, v)$

The nonvortical electric field can be expressed as the gradient of a scalar potential:

$\underline{\boldsymbol{e}}_{v}(u, v)=-\nabla \Phi(v)$

The equipotential lines in the emitter aperture are identical to the parameter lines for constant $v$ as shown in Fig. 1. On the assumption of a charge-free dielectric inside the radiator, as well as a constant permittivity $\varepsilon_{r}$, we find Laplace's equation in Bipolar coordinates (see Murray, 1979) as follows:

$\nabla^{2} \Phi(v)=\frac{(\cosh (v)-\cos (u))^{2}}{a^{2}} \cdot \frac{\partial^{2} \Phi(v)}{\partial v^{2}}=0$

The variable $a$ characterises the pole position of the coordinate system (see Fig. 1a). If Eq. (4) is integrated twice, one gets the scalar potential, which with the boundary conditions $\Phi\left(v=v_{1}\right)=0$ and $\Phi\left(v=v_{2}\right)=U_{0}$ can be expressed as:

$\Phi(v)=\frac{U_{0}}{v_{2}-v_{1}} \cdot\left(v-v_{1}\right)$

Substituting Eqs. (5) into (3), the electric field in the emitter aperture can be given by:

$\underline{\boldsymbol{E}}=A \cdot \frac{1}{a} \cdot(\cosh (v)-\cos (u)) \cdot e^{-j \cdot k \cdot z} \cdot \boldsymbol{u}_{v}$

From this the magnetic field can be calculated with application of Maxwell's second equation as follows:

$\underline{\boldsymbol{H}}=A \cdot \frac{1}{a}-$

$\cdot \sqrt{\varepsilon_{\text {emitter }} / \mu_{\text {emitter }}} \cdot(\cosh (v)-\cos (u)) \cdot e^{-j \cdot k \cdot z} \cdot \boldsymbol{u}_{u}$

In Eqs. (6) and (7), $A$ represents an amplitude factor. In addition the propagation constant $\underline{\gamma}_{z}$ was replaced by:

$\underline{\gamma}_{z}=j \cdot k=j \cdot \omega \cdot \sqrt{\mu_{\text {emitter }} \cdot \varepsilon_{\text {emitter }}}$

In order to calculate the near field characteristics, the field distribution of the emitter aperture is used to obtain the

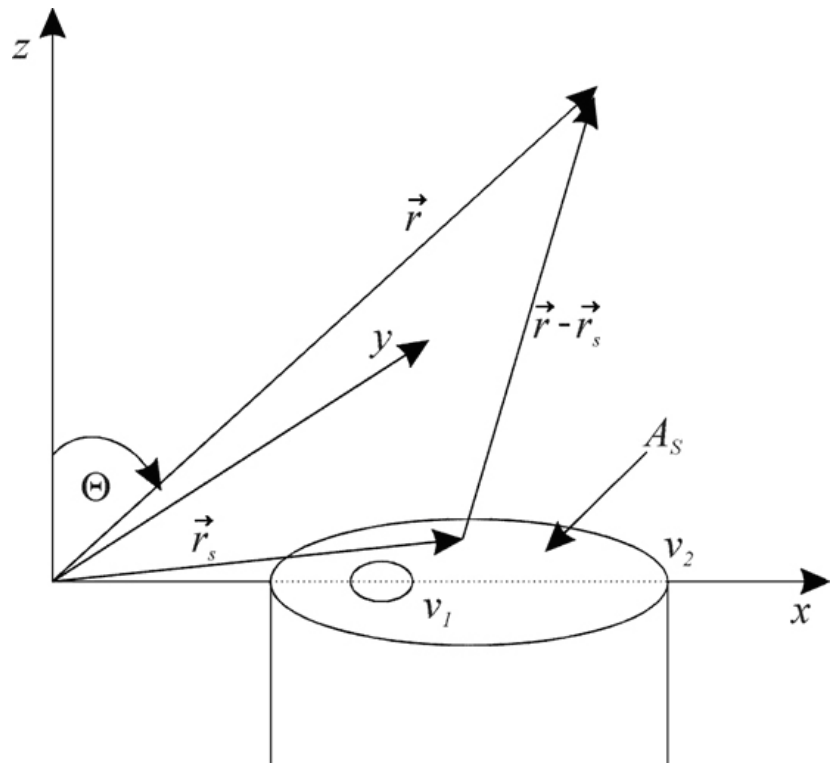

Fig. 2. Emitter arrangement in Cartesian coordinates.

equivalent electric and magnetic current densities, according to Huygen's principle:

$$
\begin{aligned}
& \underline{\boldsymbol{J}}_{\ddot{a} q}=\boldsymbol{u}_{z} \times \underline{\boldsymbol{H}}_{t} \\
& \underline{\boldsymbol{J}}_{m \ddot{a} q}=-\boldsymbol{u}_{z} \times \underline{\boldsymbol{E}}_{t}
\end{aligned}
$$

The transversal field strength results from the interference of an incident and reflected wave component. The reflection factor at the transition "radiator $\leftrightarrow$ homogenous tissue" is defined as $\Gamma$. Now the transversal electromagnetic field components in the aperture of the contact emitter can be written as:

$\underline{\boldsymbol{E}}_{t}=\underline{E}_{v}(u, v) \cdot(1+\Gamma) \cdot \boldsymbol{u}_{v}$

$\underline{\boldsymbol{H}}_{t}=\underline{H}_{u}(u, v) \cdot(1-\Gamma) \cdot \boldsymbol{u}_{u}$

Considering Eqs. (6) and (7), the equivalent current densities can be determined from the following equations:

$$
\begin{aligned}
& \underline{J}_{\ddot{a} q}=-\frac{A \cdot(1-\Gamma)}{a \cdot \sqrt{\mu_{\text {Strahler }} / \varepsilon_{\text {Strahler }}}} \cdot(\cosh (v)-\cos (u)) \cdot \boldsymbol{u}_{v} \\
& \underline{J}_{\text {mäq }}=\frac{A \cdot(1+\Gamma)}{a} \cdot(\cosh (v)-\cos (u)) \cdot \boldsymbol{u}_{u}
\end{aligned}
$$

\subsection{Calculation of the radiation fields of the contact emitter}

The magnetic vector potential

$\underline{\boldsymbol{A}}(r)=\frac{1}{4 \cdot \pi} \cdot \iint_{A_{s}} \frac{\underline{\boldsymbol{J}}_{\ddot{a} q}\left(\boldsymbol{r}_{s}\right)}{\left|\boldsymbol{r}-\boldsymbol{r}_{s}\right|} \cdot e^{-j \cdot k \cdot\left|\boldsymbol{r}-\boldsymbol{r}_{s}\right|} \cdot d A_{s}$

and the electric vector potential

$$
\underline{\boldsymbol{F}}(r)=\frac{1}{4 \cdot \pi} \cdot \iint_{A_{s}} \frac{\underline{\boldsymbol{J}}_{m \ddot{a} q}\left(\boldsymbol{r}_{s}\right)}{\left|\boldsymbol{r}-\boldsymbol{r}_{s}\right|} \cdot e^{-j \cdot k \cdot\left|\boldsymbol{r}-\boldsymbol{r}_{s}\right|} \cdot d A_{s}
$$



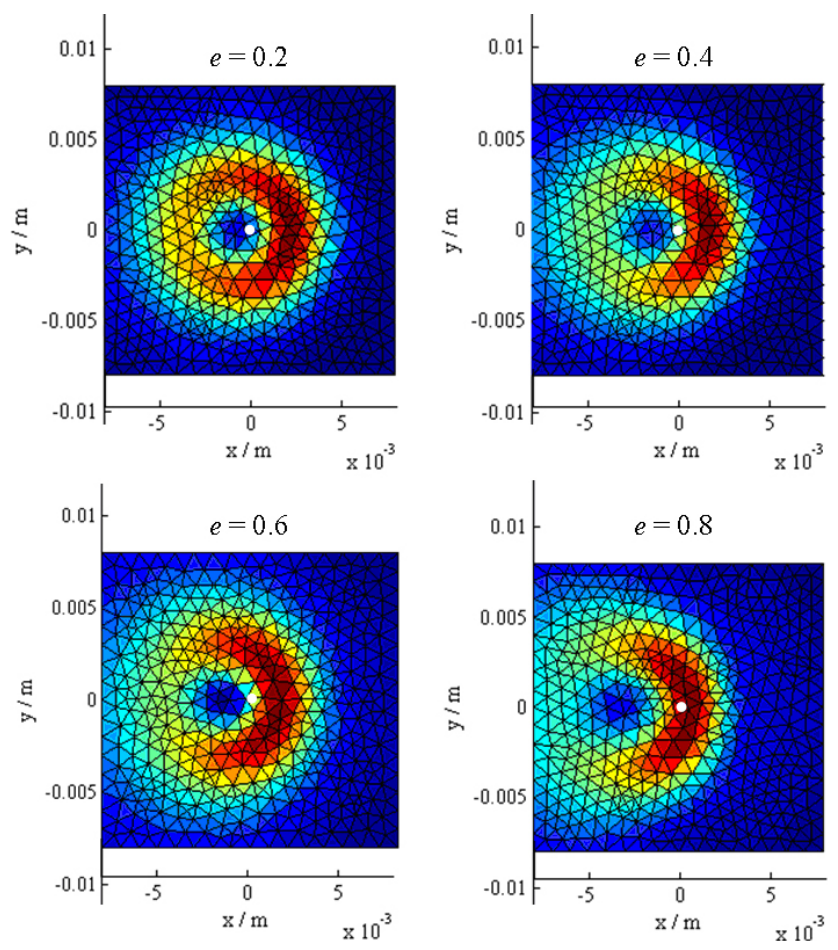

Fig. 3. Distribution of the real part of Poynting's vector in the near field as a function of the inner conductor's eccentricity factor $e$ (theory).

can be evaluated by integration over the emitter cross section $A_{S}$. The radiation field follows from:

$\underline{\boldsymbol{E}}=-\operatorname{rot} \underline{\boldsymbol{F}}+\frac{1}{j \cdot \omega \cdot \varepsilon_{\text {Gewebe }}} \cdot \operatorname{rot} \operatorname{rot} \underline{\boldsymbol{A}}$

$\underline{\boldsymbol{H}}=\operatorname{rot} \underline{\boldsymbol{A}}+\frac{1}{j \cdot \omega \cdot \mu_{\text {Gewebe }}} \cdot \operatorname{rot} \operatorname{rot} \underline{\boldsymbol{F}}$

It is not appropriate to put the origin of the coordinate system into the emitter centre for a simple organization of the integrals (see Fig. 2). The components of the vector potentials are represented in Cartesian coordinates. This facilitates the curl formation regarding the field calculation which is executed later and serves a clear near field representation. The transformation of the Bipolar unit vectors into Cartesian unit vectors gives the magnetic vector potential and the electric potential in Eqs. (16) and (17) using the substitutions $S(v, u)=\frac{\sinh (v) \cdot \sin (u)}{(\cosh (v)-\cos (u))^{2}}$ and $C(v, u)=\frac{\cosh (v) \cdot \cos (u)-1}{(\cosh (v)-\cos (u))^{2}}$.

$\underline{A}=$

$$
\begin{aligned}
& -\frac{A \cdot a \cdot(1-\Gamma)}{4 \cdot \pi \cdot \sqrt{\mu_{\text {Strahler }} / \varepsilon_{\text {Strahler }}}} \cdot \int_{0}^{2 \cdot \pi} \int_{v_{1}}^{v_{2}}\left\{C(v, u) \cdot \frac{e^{-j \cdot k \cdot\left|\boldsymbol{r}-\boldsymbol{r}_{s}\right|}}{\left|\boldsymbol{r}-\boldsymbol{r}_{s}\right|} \cdot \boldsymbol{u}_{x}+\ldots\right. \\
& \left.S(v, u) \cdot \frac{e^{-j \cdot k \cdot\left|\boldsymbol{r}-\boldsymbol{r}_{s}\right|}}{\left|\boldsymbol{r}-\boldsymbol{r}_{s}\right|} \cdot \boldsymbol{u}_{y}\right\} \cdot d v \cdot d u \\
& \underline{\boldsymbol{F}}=\frac{A \cdot a \cdot(1+\Gamma)}{4 \cdot \pi} \cdot \int_{0}^{2 \cdot \pi} \int_{v_{1}}^{v_{2}}\left\{S(v, u) \cdot \frac{e^{-j \cdot k \cdot\left|\boldsymbol{r}-\boldsymbol{r}_{s}\right|}}{\left|\boldsymbol{r}-\boldsymbol{r}_{s}\right|} \cdot \boldsymbol{u}_{x}-\ldots\right. \\
& \left.C(v, u) \cdot \frac{e^{-j \cdot k \cdot \boldsymbol{r}-\boldsymbol{r}_{s} \mid}}{\left|\boldsymbol{r}-\boldsymbol{r}_{s}\right|} \cdot \boldsymbol{u}_{y}\right\} \cdot d v \cdot d u
\end{aligned}
$$

The integral Eqs. (16) and (17) are not analytically solvable. Therefore the curl of the integrals is determined beforehand. Afterwards, a pure numeric calculation of the electric and magnetic fields is executed. For calculating the curl of a vector, it is necessary to determine the partial derivatives. Analysing the electric and magnetic vector potential, these partial derivatives are limited to the following expression:

$$
\begin{aligned}
& \underline{P}(x, y, z)=\frac{e^{-j \cdot k \cdot\left|\boldsymbol{r}-\boldsymbol{r}_{s}\right|}}{\left|\boldsymbol{r}-\boldsymbol{r}_{s}\right|}=\ldots \\
& \frac{e^{-j \cdot k \cdot \sqrt{\left(x-\frac{a \cdot \sinh (v)}{\cosh (v)-\cos (u)}\right)^{2}+\left(y-\frac{a \cdot \sin (u)}{\cosh (v)-\cos (u)}\right)^{2}+z^{2}}}}{\sqrt{\left(x-\frac{a \cdot \sinh (v)}{\cosh (v)-\cos (u)}\right)^{2}+\left(y-\frac{a \cdot \sin (u)}{\cosh (v)-\cos (u)}\right)^{2}+z^{2}}}
\end{aligned}
$$

According to Eq. (9), the vector of source point $\boldsymbol{r}_{s}$ is represented in Bipolar coordinates. The partial derivatives can be abbreviated by

$\underline{P}_{\eta}=\frac{\partial \underline{P}(x, y, z)}{\partial \eta}, \quad \underline{P}_{\eta, \tau}=\frac{\partial \underline{P}(x, y, z)}{\partial \eta \partial \tau}$

where $\eta, \tau \in\{x, y, z\}$.

Using the mentioned expressions, the following terms result from simple and double curl formation:

$$
\begin{aligned}
& \operatorname{rot} \underline{\boldsymbol{A}}=-\frac{A \cdot a \cdot(1-\Gamma)}{4 \cdot \pi \cdot \sqrt{\mu_{\text {Strahler }} / \varepsilon_{\text {Strahler }}}} \cdot \ldots \\
& \quad \int_{0}^{2 \cdot \pi} \int_{v_{1}}^{v_{2}}\left\{\left[-S(v, u) \cdot \underline{P}_{z}\right] \cdot \boldsymbol{u}_{x}+\left[C(v, u) \cdot \underline{P}_{z}\right] \cdot \boldsymbol{u}_{y}+\ldots\right. \\
& \left.\left[S(v, u) \cdot \underline{P}_{x}-C(v, u) \cdot \underline{P}_{y}\right] \cdot \boldsymbol{u}_{z}\right\} \cdot d v \cdot d u \\
& \operatorname{rot} \underline{\boldsymbol{F}}=\frac{A \cdot a \cdot(1+\Gamma)}{4 \cdot \pi} \cdot \ldots \\
& \quad \int_{0}^{2 \cdot \pi} \int_{v_{1}}^{v_{2}}\left\{\left[C(v, u) \cdot \underline{P}_{z}\right] \cdot \boldsymbol{u}_{x}+\left[S(v, u) \cdot \underline{P}_{z}\right] \cdot \boldsymbol{u}_{y}-\ldots\right. \\
& \left.\left[C(v, u) \cdot \underline{P}_{x}+S(v, u) \cdot \underline{P}_{y}\right] \cdot \boldsymbol{u}_{z}\right\} \cdot d v \cdot d u
\end{aligned}
$$

$\operatorname{rot} \operatorname{rot} \underline{A}=$

$$
\begin{aligned}
& -\frac{A \cdot a \cdot(1-\Gamma)}{4 \cdot \pi \cdot \sqrt{\mu_{\text {Strahler }} / \varepsilon_{\text {Strahler }}}} \cdot \ldots \\
& \int_{0}^{2 \cdot \pi} \int_{v_{1}}^{v_{2}}\left\{\left[S(v, u) \cdot \underline{P}_{x y}-C(v, u) \cdot \underline{P}_{y y}-C(v, u) \cdot \underline{P}_{z z}\right](21)\right. \\
& \cdot \boldsymbol{u}_{x}+\ldots\left[-S(v, u) \cdot \underline{P}_{z z}-S(v, u) \cdot \underline{P}_{x x}+C(v, u) \cdot \underline{P}_{y x}\right] \cdot \boldsymbol{u}_{y} \\
& \operatorname{rot} \operatorname{rot} \underline{\boldsymbol{F}}=\frac{A \cdot a \cdot(1+\Gamma)}{4 \cdot \pi} \cdot \ldots \\
& \int_{0}^{2 \cdot \pi} \int_{v_{1}}^{v_{2}}\left\{\left[-C(v, u) \cdot \underline{P}_{x y}-S(v, u) \cdot \underline{P}_{y y}-S(v, u) \cdot \underline{P}_{z z}\right] \cdot \boldsymbol{u}_{x}+\ldots\right. \\
& {\left[-C(v, u) \cdot \underline{P}_{z z}+C(v, u) \cdot \underline{P}_{x x}+S(v, u) \cdot \underline{P}_{y x}\right] \cdot \boldsymbol{u}_{y}+\ldots} \\
& \left.\left[S(v, u) \cdot \underline{P}_{z x}-C(v, u) \cdot \underline{P}_{z y}\right] \cdot \boldsymbol{u}_{z}\right\} \cdot d v \cdot d u
\end{aligned}
$$

Substituting Eqs. (20) and (21) into Eq. (14), and Eqs. (19) and (22) into Eq. (15) respectively, a pure numeric field calculation of the electric and magnetic field for defined space points $(x, y, z)$ is possible. As the solution is based on the use of the Bipolar coordinate system, the shift of the radiator's aperture from the origin has to be considered prior to selection of the xcoordinate. 

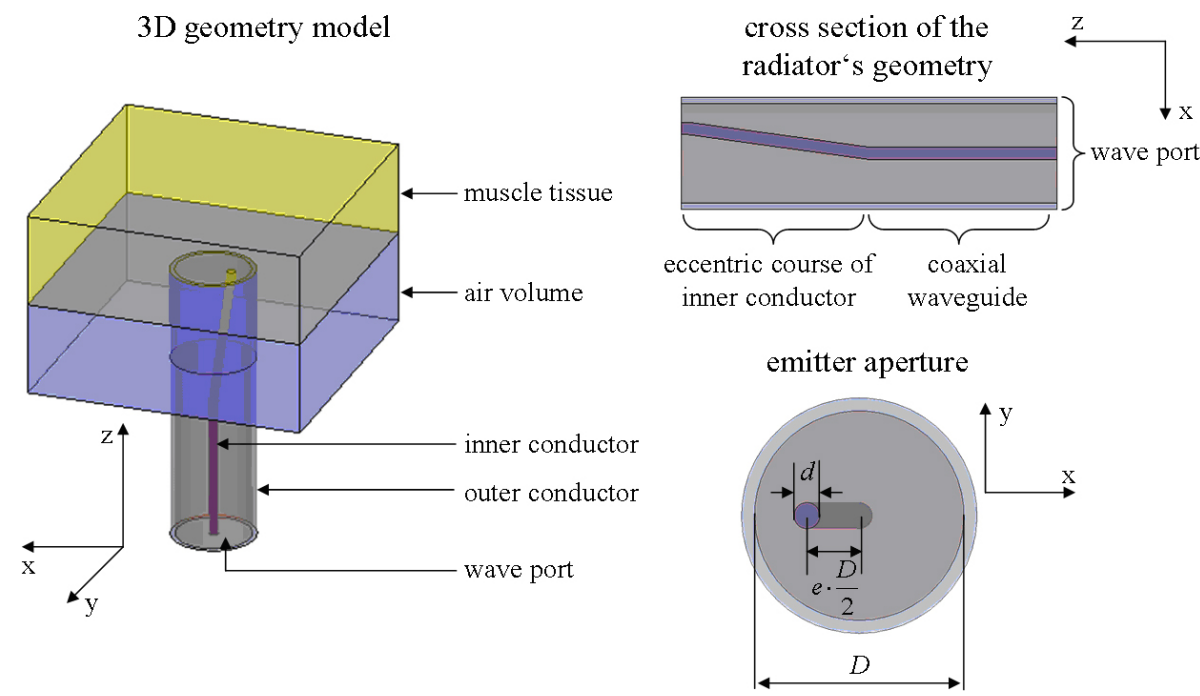

Fig. 4. Ansoft HFSS simulation model of the contact emitter.

\subsection{Graphical representation of the near field}

The programme system MATLAB is applied for a mathematical evaluation of the formalism presented above. The numerical results constitute the basis for a graphical near field representation of the radiator as a function of the eccentricity factor $e$ of the inner conductor. In Fig. 3, the real part of the complex Poynting vector is presented for defined space points in a plane parallel to the emitter aperture at a frequency of $f=10 \mathrm{GHz}$. This time-independent quantity ensures a direct comparison with the simulation results. The calculated field strength values are normalized to the maximum value of the field strength at each examined eccentricity factor $e$. The amplitudes are subdivided linearly in colour tones with increasing power density from blue to red. The square plane has an edge length of $l=16 \mathrm{~mm}$ and is placed $\Delta z=5 \mathrm{~mm}$ in front of the emitter aperture. The inner diameter of the outer conductor and the outer diameter of the inner conductor are set to $D=10 \mathrm{~mm}$ and $d=1 \mathrm{~mm}$. The relative dielectric constant inside the radiator is selected to $\varepsilon_{r \text {, waveguide }}=1$. The relative dielectric constant of the homogenous tissue in front of the emitter aperture is assigned a value of $\varepsilon_{r, t i s s u e}=50$. This is a typical value found in literature as a crude approximation for muscle tissue (see Stauffer, et al., 1998; Rossetto, et al., 2000). It can be seen clearly by Fig. 3, that the absolute maximum of the power density shifts as a function of the eccentricity factor $e$ of the inner conductor. With increasing misalignment of the inner conductor to the left-hand side of the emitter centre, the maximum moves towards the centre of the emitter aperture, which is marked by a white circle in the graphical representations.

\section{Comparison of analytical results and simulation data}

To simulate the radiation fields of the contact emitter, the commercially available simulation programme HFSS (High Frequency Stucture Simulator) from Ansoft Inc. is used. Ansoft HFSS applies the finite element method (FEM) in order to analyse the electromagnetic behaviour of complex three dimensional model geometries. The implemented FEM allows accounting for the influence of arbitrary material surrounding the antenna aperture. The FEM directly calculates the field distribution inside a defined simulation volume which is confined by a radiation boundary for calculating far fields. The construction of the radiator's simulation model is summarised in Fig. 4. In contrast to the theoretical analysis, the simulation with Ansoft HFSS considers higher order modes inside the coaxial waveguide with an eccentric course of the inner conductor and in the emitter aperture. The simulation models used in the following are based on the geometry and material dates given under Sect. 2.

\subsection{Distribution of power density in the near field area}

The results of the near field simulation are shown in Fig. 5. The real part of the complex Poynting vector is calculated for defined space points of the above mentioned plane, as a function of the eccentricity factor $e$ of the inner conductor. Comparing the graphical representation with those in Fig. 3 it is obvious that the analytical and the simulation results correspond quite well. But the influence of the higher order modes neglected in the analytic approach is also obvious. With increasing eccentricity factor $e$ a second maximum appears. This area of high power density is located at the left boundary of the outer conductor. So there is no relevance for the medical application considered in this paper because only very small superficial skin areas of cancerous tissue or 

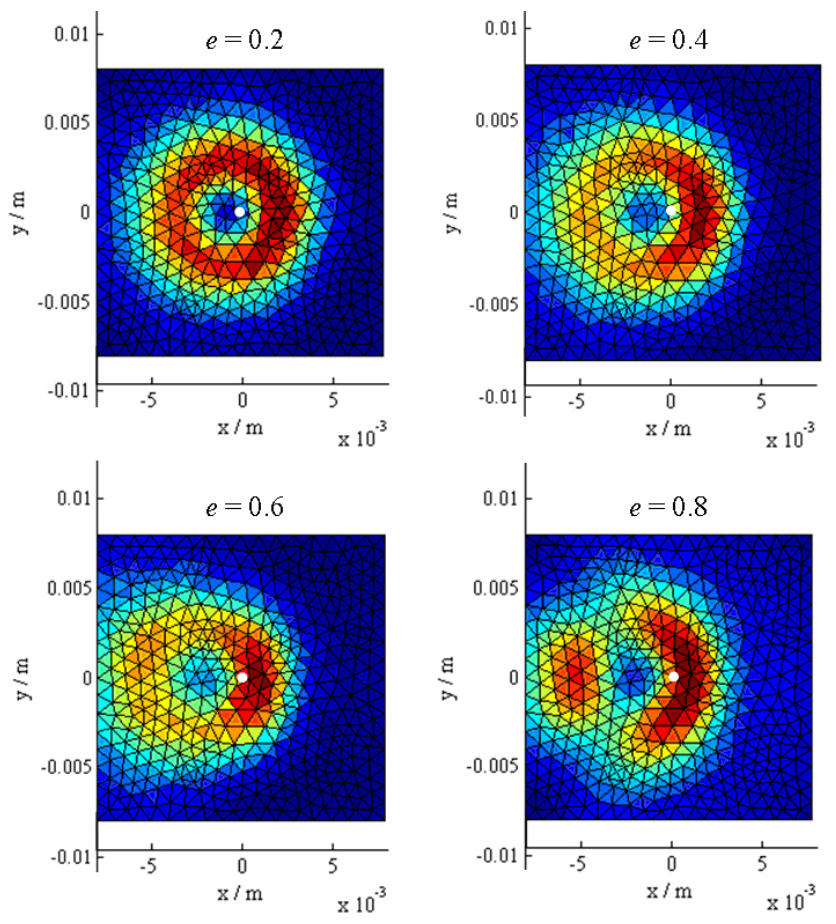

Fig. 5. Simulated distribution of the real part of Poynting's vector in the near field as a function of the inner conductor's eccentricity factor $e$.

biological active points interacting with the main maximum are considered. In comparison with the analytic results the deviating field distribution is due to the higher order modes. But the overall effect of positioning the absolute maximum of power density at the centre of the contact emitter by an eccentric shift of the inner conductor is confirmed by the simulation results as well.

\subsection{Far field characteristics}

In this section the differences in far field characteristics caused by ignoring the effects of higher order modes in the analytical approach in comparison to the simulation are investigated. Therefore Fig. 6 contains the far field plots and spherical far field cuts to clarify these differences. Analysis and simulation are performed with an eccentricity factor $e=0.5$ at $f=10 \mathrm{GHz}$. In contrast to the analytical dates the simulation results describe an antenna with a maximum at $\Phi=0^{\circ}$ and $\Theta=335^{\circ}$. However the theoretical approach predicts a nearly constant power density surrounding a global minimum in the direction of the contact emitter's centre. The theory based radiation pattern predict maxima at $\Phi=90^{\circ}$, $\Theta=30^{\circ}$ and $\Phi=90^{\circ}, \Theta=330^{\circ}$, which are situated pointsymmetrically to the focal point of the respective directivity pattern. The distinct minimum in front of the aperture, which is typical for the rotationally symmetric radiation pattern of a coaxial radiator $(e=0)$, also appears in the simulation results at the same position. (a)
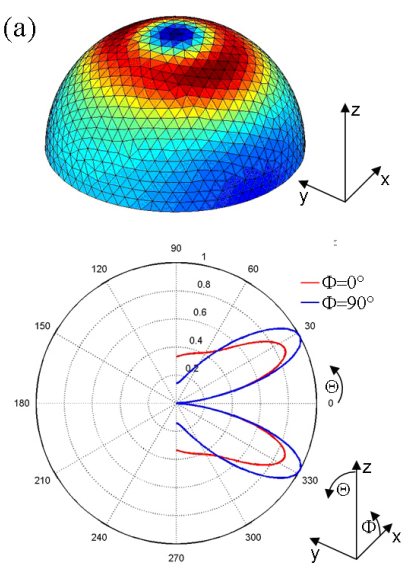

(b)
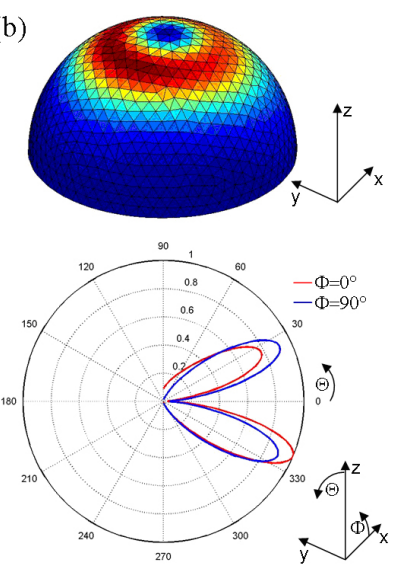

Fig. 6. Radiation patterns and directivity diagrams from analytical computation (a) and simulation (b) based on an eccentricity factor $e=0.5$.

\section{Conclusions}

The thought experiment for the eccentric shift of the inner conductor is confirmed by the theoretical analysis, as well as by the simulation results of Ansoft HFSS. Due to the neglect of the higher order modes with the theoretical analysis, the results differ slightly in the near field area. Nevertheless the simulation results of the near field are verified by the theoretical analysis in spite of exclusive consideration of the fundamental TEM mode. It could be shown unambiguously that the position of the maximum temporal average of Poynting's vector, is a function of the inner conductor's eccentricity factor $e$ in the emitter aperture. Depending on the geometry and material dates, an eccentric position of the inner conductor could be determined, so that the maximum of the power density is localized directly above the emitter centre in the near field area. The simulated far field radiation patterns differ from the analytically calculated results mainly in the position of the maxima. A better agreement between the simulation data and the analytical results can only be obtained by an expansion of the proposed analytical solution to consider for the higher order modes in the emitter aperture.

\section{References}

Bakhtiari, S., Ganchev, S. I., and Zoughi, R.: Analysis of radiation from open-ended coaxial line into stratified dielectrics, IEEE Transaction on Microwave Theory and Techniques, 42, 12611267, 1994.

Kalantaewskaja, K. A.: Morphologie und Physiologie des Hautgewebes, Moskau, Medizin-Verlag, 1972.

Maloney, J. G., Smith, G. S., and Scott, W. R.: Accurate computation of the radiation from simple antennas using the finitedifference time-domain method, IEEE Transactions on Antennas and Propagation, 38, 1059-1068, 1990. 
Mosig, J. R., Besson, J. C. E., Gex-Fabry, M., and Gardiol, F. E.: Reflektion of an open-ended coaxial line and applikation to nondestruktive measurement of materials, IEEE Transactions on Instrumentation and Measurement, 30, 46-51, 1981.

Murray, R.: Handbuch für Mathematik, New York, McGraw - Hill, 1979.

Nevels, R. D., and Wheeler, J. E.: Radiation from a dielectric coated hemispherical conductor fed by a coaxial transmission line, IEEE Transactions on Electromagnetic Compatibility, 31, 16-20, 1989.

Rossetto, F., Diederich, C. J., and Stauffer, P.: Thermal an SAR characterization of multielement dual concentric conductor microwave applicators for hyperthermia, a theoretical investigation, Medical Physics, 27, 4, 2000.

Stauffer, P., Rossetto, F., Leoncini, M., and Gentilli, G. B.: Radiation Patterns of Dual Concentric Conductor Microstrip Antennas for Superficial Hyperthermia, IEEE Transaction on Biomedical Engineering, 45, 5, 1998.
Stuchly, M. A. and Stuchly, S. S.: Coaxial line reflection methods for measuring dielectric properties of biological substances at radio and microwave frequencies - a review, IEEE Transactions on Instru-mentation and Measurement, 29, 176-183, 1980.

Stuchly, M. A., Brady, M. B., Stuchly, S. S., and Gajda, G. B.: Equivalent circuit of open-ended coaxial line in a lossy dielectric, IEEE Transactions on Instrumentation and Measurement, 31, 116-119, 1982.

Sumbar, E., Chute, F. S., and Vermeulen, F. E.: Implementation of radiation boundary conditions in the finite element analysis of eletromagnetic wave propagation, IEEE Transactions on Microwave Theory and Techniques, 39, 267-273, 1991. 